\title{
Acetylcholinesterase Reactivators (HI-6, Obidoxime, Trimedoxime, K027, K075, K127, K203, K282): Structural Evaluation of Human Serum Albumin Binding and Absorption Kinetics
}

\section{Filip Zemek $^{1{ }^{1} *}$, Jana Karasova Zdarova ${ }^{1}$, Vendula Sepsova ${ }^{1}$ and Kamil Kuca ${ }^{1,2,3}$}

1 Faculty of Military Health Sciences, University of Defence, Trebesska 1575, Hradec Kralove 500 01, Czech Republic; E-Mails: karasova@pmfhk.cz (J.K.Z.); sepsova@pmfhk.cz (V.S.); kamil.kuca@fnhk.cz (K.K.)

2 Biomedical Research Centre, University Hospital, Sokolska 581, Hradec Kralove 500 05, Czech Republic

3 Department of Chemistry, Faculty of Science, University of Hradec Kralove, Rokitanskeho 62, Hradec Kralove 500 03, Czech Republic

* Author to whom correspondence should be addressed; E-Mail: zemek.filip@gmail.com; Tel.: +420-777-069-894.

Received: 27 June 2013; in revised form: 23 July 2013 / Accepted: 25 July 2013 /

Published: 2 August 2013

Abstract: Acetylcholinesterase (AChE) reactivators (oximes) are compounds predominantly targeting the active site of the enzyme. Toxic effects of organophosphates nerve agents (OPNAs) are primarily related to their covalent binding to AChE and butyrylcholinesterase (BChE), critical detoxification enzymes in the blood and in the central nervous system (CNS). After exposure to OPNAs, accumulation of acetylcholine (ACh) overstimulates receptors and blocks neuromuscular junction transmission resulting in CNS toxicity. Current efforts at treatments for OPNA exposure are focused on non-quaternary reactivators, monoisonitrosoacetone oximes (MINA), and diacylmonoxime reactivators (DAM). However, so far only quaternary oximes have been approved for use in cases of OPNA intoxication. Five acetylcholinesterase reactivator candidates (K027, K075, K127, K203, K282) are presented here, together with pharmacokinetic data (plasma concentration, human serum albumin binding potency). Pharmacokinetic curves based on intramuscular application of the tested compounds are given, with binding information and an evaluation of structural relationships. Human Serum Albumin (HSA) binding studies have not yet been performed on any acetylcholinesterase reactivators, and correlations 
between structure, concentration curves and binding are vital for further development. HSA bindings of the tested compounds were 1\% (HI-6), 7\% (obidoxime), $6 \%$ (trimedoxime), and $5 \%, 10 \%, 4 \%, 15 \%$, and $12 \%$ for K027, K075, K127, K203, and K282, respectively.

Keywords: acetylcholinesterase; oximes; human serum albumin; pharmacokinetics; reactivator; antidote; nerve agent

\section{Introduction}

Drug development is challenging work with numerous unknown variables that have to be taken into account, including the detailed pharmacokinetics of novel compounds [1]. Here, we present in vitro and in vivo determinations of plasma concentration and human serum albumin (HSA) binding potency of newly synthesized acetylcholinesterase (AChE, E.C. 3.1.1.7) reactivators. For newly synthesized compounds, properties of absorption, distribution, metabolism and excretion ("ADME") are critical for determining the future potency of compounds in clinical practice. Indeed, the "Lipinski rule of 5" quantifies the properties that compounds should possess to be eligible for success [2,3]. This rule postulates that poor absorption or permeation is more likely when there are more than $5 \mathrm{H}$-bond donors, $10 \mathrm{H}$-bond acceptors, the molecular weight is greater than 500 and the calculated $\log P$ is greater than 5. Lipinsky et al. (1997) [2] stated these properties for drug candidates to be used orally, however it can be more or less applied to all drug formulations with appropriate modifications.

Many years before Lipinsky postulated his ADME properties, Sudlow et al. (1975) [4] found that HSA interacts with various compounds, and defined two major binding sites responsible in the majority of cases for altering the pharmacokinetic profile of exogenous compounds. The two binding sites were named site I and site II, or Sudlow sites I and II. In addition to these two sites, other less potent binding regions have been identified: e.g., domain III (sub-domain B) for thyroxine, propofol; domain II (sub-domain B) for halothane, ibuprofen; domain I (sub-domain B) for indomethacin, azapropazone. It is important to note that substances can bind to several sites with different affinities $[5,6]$. Compounds that bind to site I have several structural similarities. Most ligands seem to be dicarboxylic acids and/or have heterocyclic molecules with a negative charge localized in the middle of the molecule. Since structurally different compounds (e.g., bilirubin and warfarin) bind to the same site, this points to the flexibility and adaptability of the Sudlow I site [7-11]. Site II, or Sudlow II, is also known as the indole-benzodiazepine site, according to the ligands with the highest affinity. In general, ligands for this site are often aromatic carboxylic acids with a negatively charged acidic group away from the hydrophobic center, e.g., non-steroidal anti-inflammatory drugs (NSAIDs). Compared to site I, site II is smaller (narrower) and less flexible, which is reflected by the absence of large ligands binding to site II (e.g., bilirubin, hemin, hematin or other porphins) [12-17].

Our work focuses on the pharmacokinetic properties of antidotes for irreversible AChE inhibitors that act directly on the cholinergic system and lead to hyperactivation of the choline system with all accompanying negative symptoms, e.g., bradycardia, hypotension, hypersecretion, bronchoconstriction, GI tract hypermotility, which, if left untreated, lead to death [18]. Furthermore, the simple structure of these inhibitors and their relatively easy and inexpensive synthesis makes them even more dangerous, 
since it makes them easy to be acquired by terrorist groups and misused against civilian personnel (e.g., sarin, tabun, soman, VX) [19]. However, numerous pesticides (parathion, malathion, methyl parathion, chlorpyrifos, diazinon etc.) used every day by farmers around the developing world are also inhibitors of $\mathrm{AChE}$ and intoxication is unfortunately very common [20].

In the work presented here, oximes (HI-6, obidoxime, trimedoxime, Figure 1) that are structurally and therapeutically well-defined were used as standards to help correlate the biological behavior of five newly synthetized compounds (K027, K075, K127, K203, K282, Figure 2). Some pharmacokinetic data of HI-6, obidoxime, trimedoxime, K027, and K203 have been published earlier [21-24], but binding to human serum albumin and structural correlations have not been performed before on these compounds, and using a whole set of promising structurally heterogonous AChE reactivators make comparisons more robust. Novel compounds are structural analogues combining features from all three established oximes. In vitro tests have confirmed improved reactivation efficacy [25,26], so detailed pharmacokinetic data are needed for further testing [27].

Figure 1. Structures of the standard Acetylcholinesterase (AChE) reactivators used.

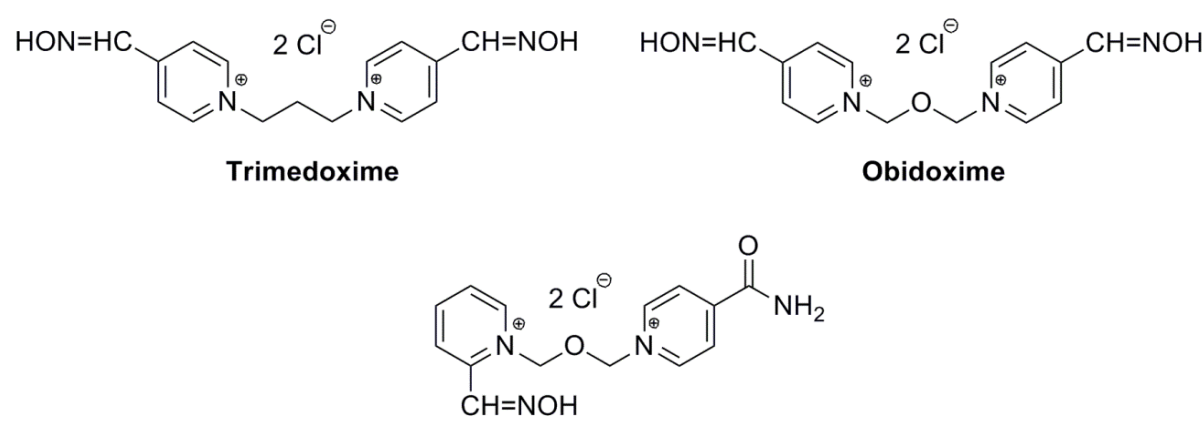

HI-6

Figure 2. Novel AChE reactivators tested in this study.<smiles></smiles><smiles></smiles><smiles>[R17]CC[n+]1ccc(C=N)cc1</smiles><smiles></smiles><smiles>N=Cc1cc[n+](C/C=C\C[n+]2ccc(C=N)cc2)cc1</smiles>

Recently, novel hypotheses and compounds with unique structures have been introduced as potential antidotes against OPNA poisoning (MINA, DAM, non-quaternary oximes, bioscavengers, etc.). Nevertheless, more experimental work needs to be conducted to demonstrate the superiority of these new approaches. Currently, quaternary oximes are still the only compounds approved for this use [28-30]. 
Though based on the known information and structures of the tested compounds significant binding cannot be expected, we here provide essential experimental confirmation.

\section{Results and Discussion}

\subsection{Tolerability}

After i.m. application of the tested compounds, no side effects were observed the experimental animals. Moreover, no signs of discomfort such as pain or convulsion of the hind limb muscles were observed during the experimental $240 \mathrm{~min}$ or the follow-up period. The follow-up period was conducted 24 and $48 \mathrm{~h}$ after the last application. None of the animals showed any convulsions or movement difficulty. Eating and drinking habits were normal.

\subsection{Plasma Concentrations}

Obtained $C_{\max }$ data from the experiments conducted on male Wister rats are given in Table 1 . Kinetic curves where $C_{\max }$ can be linked to the relevant time interval. HI-6 and trimedoxime exhibit slow elimination from the blood and thereby are present longer in higher concentrations. In contrast, obidoxime has a quick onset and also relatively fast elimination. The kinetic curves of K127, K075 and K282 show even longer elimination periods and relatively stable concentrations over longer periods of time. The kinetic curves of K075 and K282 are quite similar, with both graphs showing a less pronounced curve and missing the characteristic elimination profile, and thus most resemble the profile of trimedoxime. K027 and K203 exhibit a kinetic profile with a relatively well-defined peak determining $C_{\max }$. These curves can be considered analogous to those of HI-6, and even $C_{\max }$ values are comparable. Obidoxime seems to have a unique profile with a relatively swift elimination phase and the highest $C_{\max }$ peak.

Table 1. $C_{\max }$ of i.m. injected AChE reactivators.

\begin{tabular}{cc}
\hline AChE Reactivators & Plasma Concentration $\left(\boldsymbol{C}_{\max } \boldsymbol{\mu g} / \mathbf{m L}\right)$ \\
\hline HI-6 & $15.26 \pm 1.71$ \\
Obidoxime & $22.76 \pm 4.28$ \\
Trimedoxime & $16.64 \pm 4.25$ \\
K027 & $17.61 \pm 1.60$ \\
K075 & $15.50 \pm 2.82$ \\
K127 & $15.35 \pm 3.28$ \\
K203 & $16.63 \pm 5.29$ \\
K282 & $11.56 \pm 2.31$ \\
\hline
\end{tabular}

\subsection{HSA Binding}

Samples $(1 \mathrm{~mL})$ were prepared in triplicates for each compound with one control sample (HSA was substituted by phosphate buffer) and transferred into Centrifree ${ }^{\circledR}$ Ultrafiltration Devices. The acquired filtrate was subsequently analyzed according to previously published methods [31,32]. AUC from the control sample was considered as a reference value of $100 \%$. Filter retention was determined to be below $1 \%$, as declared by the manufacturer. All compounds were tested in triplicates, and the 
final AUC was the mean of three consecutive measurements and correlated with the measurements of blank samples.

To date there has only been a general consensus that oximes have no significant interactions with HSA and no binding studies had been experimentally performed. Based on the results shown in Table 2 the interaction potential of the tested compounds is low. HI-6, obidoxime, and trimedoxime, which are standardly used in the military, exhibited pharmacologically insignificant binding of $1 \%$, $7 \%$, 6\%, respectively. K127 (4\%) and K027 (5\%) exhibited the same low binding potency as the standards used. However, K075, K203, and K282 had relatively high increases in binding potency of $10 \%, 15 \%$, and $12 \%$, respectively. Nevertheless, this increase is still insignificant in terms of pharmacological properties.

Table 2. Binding of selected compounds to Human Serum Albumin (HSA).

\begin{tabular}{cc}
\hline AChE Reactivators & Binding (\%) $^{\text {a }}$ \\
\hline HI-6 & 1 \\
Obidoxime & 7 \\
Trimedoxime & 6 \\
K027 & 5 \\
K075 & 10 \\
K127 & 4 \\
K203 & 15 \\
K282 & 12 \\
\hline
\end{tabular}

${ }^{a}$ results are the mean of three independent measurements.

\subsection{Discussion}

All compounds and standards selected for testing are structurally similar, and during in vitro screening tests also had high reactivation potency. Based on these preliminary experiments further testing to obtain pharmacokinetic data was performed. We used in vivo experiments to acquire relevant kinetic data for these compounds. From Figure 3 it is clear that HI-6 and trimedoxime have an ideal pharmacokinetic profile. However, $C_{\max }$ is reached only after a relatively long time, which could be a disadvantage when rapid reactivation is needed especially against quickly aging AChE inhibitors (e.g., soman). Nevertheless, HI-6 is the only available oxime so far to show some efficacy against soman intoxication [32]. The $C_{\max }$ time delay seems to be of importance, since obidoxime reaches $C_{\max }$ swiftly when compared to the other compounds and has relatively rapid elimination from the plasma. But at the same time obidoxime has demonstrated significant potency against a great number of AChE inhibitors and is part of the military standard antidote kits. Indeed, $C_{\max }$ values alone cannot be correlated with the therapeutic efficacy of these compounds. Since these compounds serve as antidotes against nerve agents, a clinical trial determining minimum plasma concentrations needed for effective therapy is not feasible. For these reasons, characteristics of these compounds are based predominantly on pre-clinical experimental data.

As is evident from Table 3, the binding to HSA by the studied compounds is insignificant for potential clinical use, not even exceeding 20\%. Nonetheless, correlations between binding, structure and pharmacokinetic curves can be drawn. The compounds K075, K203, and K282 have the highest 
binding potency (Table 3). Furthermore, all three compounds have linking chains between the two pyridine rings of the same length $(4 \mathrm{C})$ with a double bond between $2 \mathrm{C}$ and $3 \mathrm{C}$. None of the other compounds have similar links (i.e., they either contain an oxygen atom, only 3C, or no double bond). Moreover, the pharmacokinetic curves for compounds K075, K203, and K282 highly resemble each other (Figure 4), with profiles reflecting the possibility that the rise of plasma concentrations is much slower as HSA binds a higher percentage of these compounds, and their $C_{\max }$ resemble a plateau because HSA is liberating the bound compound. Though these results from the kinetic data are intriguing, crystallographic tests would have to be performed to confirm this hypothesis.

Figure 3. Pharmacokinetic curves of the standards.
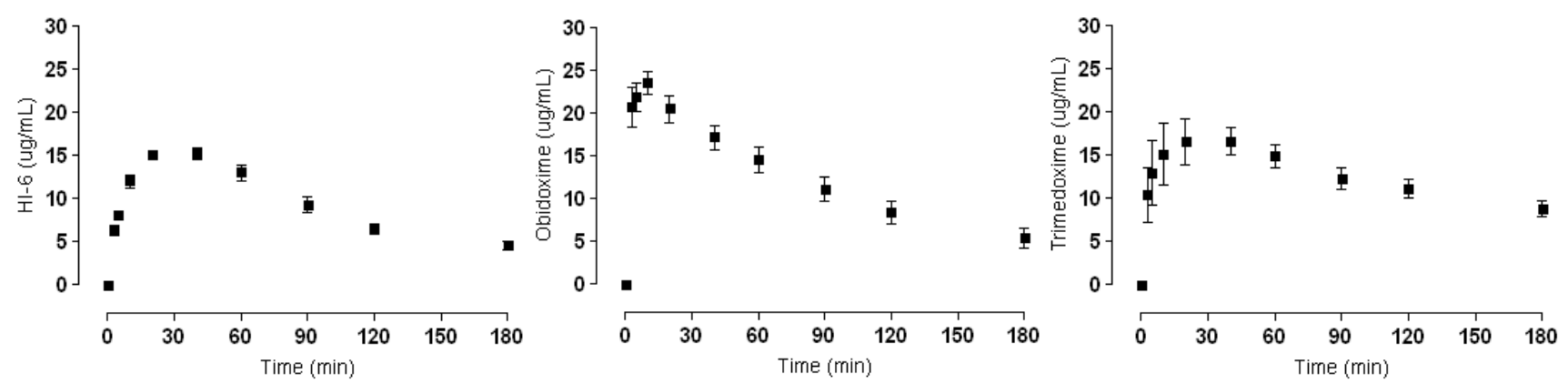

Figure 4. Pharmacokinetic curves of the tested novel oximes.
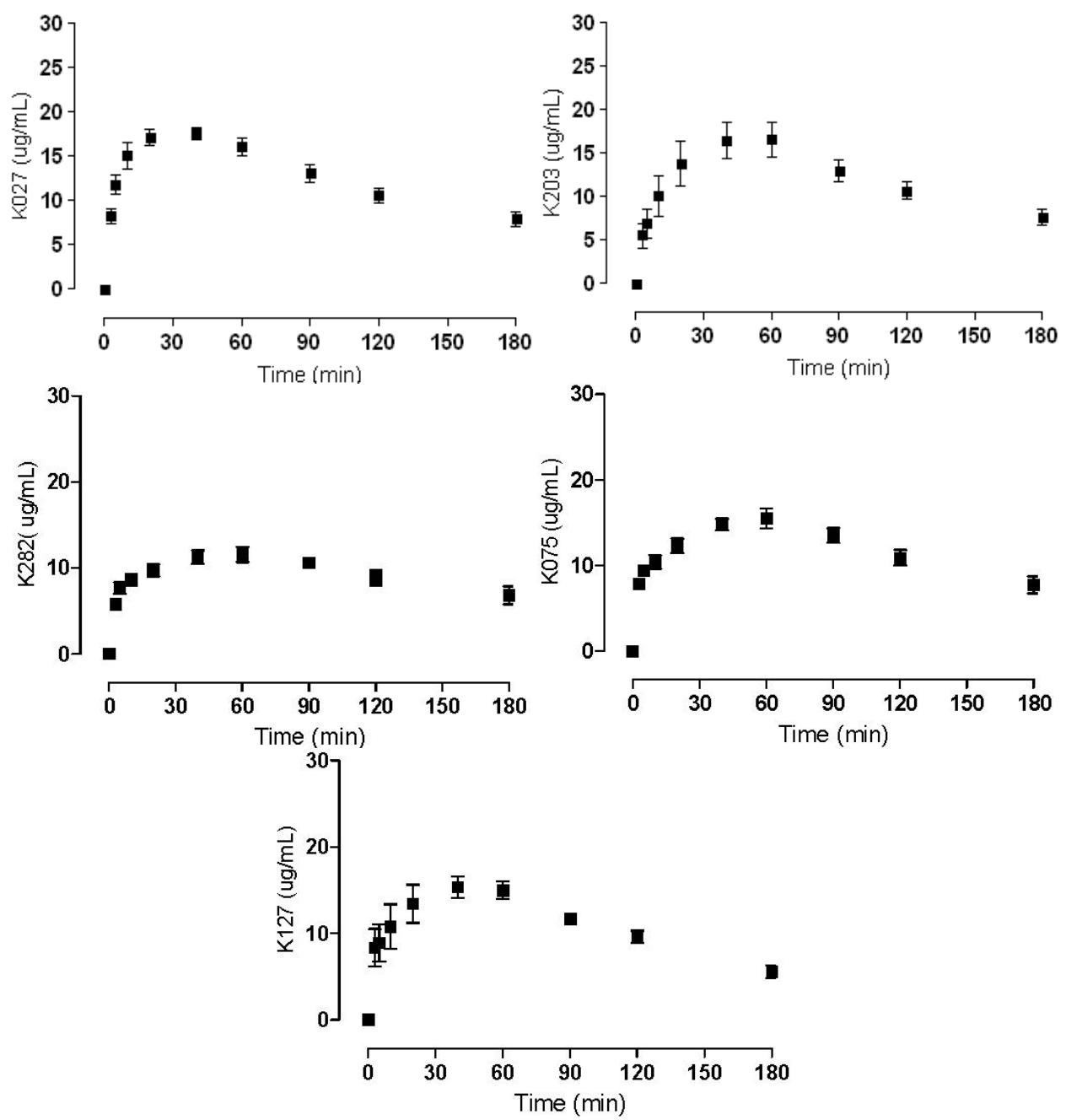


\section{Experimental Section}

\subsection{Chemicals}

Albumin, phosphate buffer saline and acetonitrile super gradient grade G Chromosolve ${ }^{\circledR}$ were purchased from Sigma-Aldrich (Prague branch, Czech Republic). Phosphoric acid (85\%) was purchased from Merck (Dermstadt, Germany). HPLC grade water was obtained by a Millipore reverse osmosis system (Goro, Prague, Czech Republic).

\subsection{Apparatus}

The HPLC system used was an Agilent 1260 Infinity Quaternary LC (Agilent Technologies Prague branch, Czech Republic) with a Coulochem II detector-analytical cell model 5011 (ESA, Bedford, MA, USA). Chemstation software (Agilent Technologies Inc., Morges, Switzerland) was used for data acquisition and interpretation.

Protein separation was performed using Centrifree ${ }^{\circledR}$ Ultrafiltration Devices (Millipore, Ireland BV, Tullagreen, Carrightwohill, Country Cork, Ireland). Ultracel regenerated cellulose membrane with a surface area $0.92 \mathrm{~cm}^{2}$ designed to retain $99.9 \%$ of serum proteins was used.

\subsection{Sample Preparation}

The HSA concentration $45 \mathrm{~g} / \mathrm{L}$ used in the binding study was derived from the average physiological concentration in a healthy population. The appropriate amount of HSA was dissolved in phosphate buffer saline and left for $15 \mathrm{~min}$ in an ultrasound basin to ensure homogenous albumin solution. Samples were prepared by pipetting $900 \mu \mathrm{L}$ of albumin solution and $100 \mu \mathrm{L}$ of phosphate buffer saline with each tested substance (plasma concentrations of the tested compounds were derived from pharmacokinetic studies described below) into Eppendorf microtubes and vortexed to ensure a homogenous distribution of the tested compound. Incubation conditions used were $90 \mathrm{~min}$ at $37{ }^{\circ} \mathrm{C}$ with continuous shaking, followed by sample transfer into Centrifree ${ }^{\circledR}$ Ultrafiltration Devices and centrifugation at $4380 \mathrm{rpm}$ for $90 \mathrm{~min}, 37^{\circ} \mathrm{C}$. The acquired filtrate was subsequently analyzed on the HPLC system by the method described further below.

\subsection{Calibration}

Calibration curves were established for all measured compounds in the concentration range of $100,75,50,25,12.56 .25$ and $0 \mu \mathrm{g} / \mathrm{L}$. All samples were prepared in triplicates and in rat plasma using exactly the same procedure as samples from the actual experiments. The rat plasma was spiked with known concentrations of measured compounds and analyzed the same day. At the end of measurements calibration was repeated to test the precision of the method.

\subsection{Pharmacokinetic Studies}

Selected plasma concentrations of tested compounds were previously determined from pharmacokinetic studies performed on male Wister rats (body weight $230 \pm 15 \mathrm{~g}$; Anlab Inc. Prague, Czech Republic). All tested animals were kept in the Vivarium at the Faculty of Military Health 
Sciences, Hradec Kralove at constant temperature $\left(22 \pm 2{ }^{\circ} \mathrm{C}\right)$, humidity $(55 \% \pm 6 \%)$, and regulated 12 $\mathrm{h}$ light-dark cycles. Standard laboratory food and tap water were available ad libitum. The experiment was performed under the supervision of the Ethical Committee of the Faculty of Military Health Sciences, University of Defence, Hradec Kralove, Czech Republic.

All animals were left to adapt for seven days in their new surrounds to eliminate the stress factor. Tested compounds were injected intramuscularly into the hind limb. Applied doses were calculated as $5 \%$ of $\mathrm{LD}_{50}$ [31,32] (tested compounds and doses applied are listed in Table 3). Before application, compounds were dissolved in a saline solution $(0.9 \% w / v \mathrm{NaCl})$ of $0.1 \mathrm{~mL} / 100 \mathrm{~g}$ of animal weight. Animals were narcotized by an intraperitoneal injection of pentobarbital $(50 \mathrm{mg} / \mathrm{kg})$. Cannulation of the arteria carotis was used for blood withdrawals; loss of blood was compensated with saline solution $(300 \mu \mathrm{L})$ via a cannula in the vena jugularis. Each blood sample was taken just before the subsequent administration in the following time intervals: 3, 5, 10, 20, 30, 40, 60, 90, 120 and 180 min after injection $(N=7$, seven animals for each compound). All animals survived. Blood samples were centrifuged at 10,000 rpm for $15 \mathrm{~min}, 10{ }^{\circ} \mathrm{C}$ (Universal 320R, Hettich, Germany. The plasma was immediately stored at $-80^{\circ} \mathrm{C}$ until $\mathrm{HPLC}$ analysis.

Table 3. List of tested compounds and applied doses.

\begin{tabular}{cc}
\hline Tested Substance & Administered Dose $\mathbf{i . m .}(\mathbf{m g} / \mathbf{k g})$ \\
\hline HI-6 & 22.23 \\
Obidoxime & 22.23 \\
Trimedoxime & 22.07 \\
K027 & 22.07 \\
K075 & 23.00 \\
K127 & 24.39 \\
K203 & 23.00 \\
K282 & 23.00 \\
\hline
\end{tabular}

\subsection{HPLC Analysis}

Before HPLC analysis, plasma samples underwent acetonitrile deproteinization (1:4; plasma/acetonitrile) followed by centrifugation at $14,000 \mathrm{rpm}$ for $15 \mathrm{~min}, 10{ }^{\circ} \mathrm{C}$. All samples were analyzed in triplicates. All analyses were done on a LiChrospher ${ }^{\circledR} 60,250 \times 4.6(5 \mu \mathrm{m})$ analytical column with a $4 \times 4$ guard column (RP-select B, Merck, Damstadt, Germany). The mobile phase was used for all compounds with slight changes to the $\mathrm{pH}$ and the ratio of acetonitrile/purified water or in the concentration of octane sulfonic acid-sodium salt. The composition of the mobile phase used was as follows 20:80 ( $v / v)$ acetonitrile/aqua purificata with octane sulfonic acid sodium salt $(6 \mathrm{mM})$. The $\mathrm{pH}$ was adjusted with phosphoric acid $\left(\mathrm{H}_{3} \mathrm{PO}_{4}\right)$ [31].

The analytical cell and guard cell had voltage set at $+350 \mathrm{mV},+650 \mathrm{mV}$ and $1000 \mathrm{mV}$, respectively. The detector gain was set at $2 \mu \mathrm{A}$ and all data were acquired at conditioned room temperature $\left(22{ }^{\circ} \mathrm{C}\right)$.

GraphPad Prism, version 5.0 (GraphPad Software, San Diego, CA, USA) was used for statistical analysis. 


\section{Conclusions}

Structure influences the pharmacokinetic properties of AChE reactivators and should be considered when designing novel compounds. Naturally, efficacy is the most important characteristic but the behavior of the compounds in the plasma is just as important. Binding to HSA even at lower concentrations can alter pharmacokinetic curves, delay the onset of $C_{\max }$ and prolong the elimination interval. It seems that a double bond in the linking chain has the potential to increase the binding affinity towards HSA and change the pharmacokinetic profile.

\section{Acknowledgments}

The authors would like to acknowledge support of the Ministry of Defence, Czech Republic: A long term organization development plan 1011.

\section{Conflict of Interest}

The authors declare no conflict of interest.

\section{References}

1. Singh, S.S. Preclinical pharmacokinetics: An approach towards safer and efficacious drugs. Curr. Drug Metab. 2006, 7, 165-182.

2. Lipinski, C.A.; Lombardo, F.; Dominy, S.W.; Feeney, P.J. Experimental and computational approaches to estimate solubility and permeability in drug discovery. Adv. Drug Deliv. Rev. 1997, 23, 3-25.

3. Walters, W.P. Going further than Lipinski's rule in drug design. Expert Opin. Drug Discov. 2012, 7, 99-107.

4. Sudlow, G.; Birkett, D.J.; Wade, D.N. The characterization of two specific drug binding sites on human serum albumin. Mol. Pharmacol. 1975, 11, 824-832.

5. Hage, D.S.; Austin, J. High-performance affinity chromatography and immobilized serum albumin as probes for drug and hormone binding. J. Chromatogr. B Biomed. Sci. Appl. 2000, 739, 39-54.

6. Kim, H.S.; Wainer, I.W. Rapid analysis of the interactions between drugs and human serum albumin (HSA) using high-performance affinity chromatography (HPAC). J. Chromatogr. B Biomed. Sci. Appl. 2008, 870, 22-26.

7. Kragh-Hansen, U. Relations between high-affinity binding sites for L-tryptophan, diazepam, salicylate and Phenol Red on human serum albumin. Biochem. J. 1983, 209, 135-142.

8. Kragh-Hansen, U. Relations between high-affinity binding sites of markers for binding regions on human serum albumin. Biochem. J. 1985, 225, 629-638.

9. Kragh-Hansen, U. Evidence for a large and flexible region of human serum albumin possessing high affinity binding sites for salicylate, warfarin, and other ligands. Mol. Pharmacol. 1988, 34, $160-171$. 
10. Joseph, K.S.; Moser, A.C.; Basiaga, S.B.G.; Schiel, J.E.S.; Hage, D.S. Evaluation of alternatives to warfarin as probes for Sudlow site I of human serum albumin: Characterization by high-performance affinity chromatography. J. Chromatogr. A 2009, 1216, 3492-3500.

11. Petersen, C.E.; Ha, C.E.; Harohalli, K.; Feix, J.B.; Bhagavan, N.V. A dynamic model for bilirubin binding to human serum albumin. J. Biol. Chem. 2000, 275, 20985-20995.

12. Ghuman, J.; Zunszain, P.A.; Peptitpas, I.; Bhattacharay, A.A.; Otagari, M.; Curry, S. Structural basis of the drug-binding specificity of human serum albumin. J. Mol. Biol. 2005, 353, 38-52.

13. Varshney, A.; Ahmad, B.; Khan, R.H. Comparative studies of unfolding and binding of ligands to human serum albumin in the presence of fatty acid: Spectroscopic approach. Int. J. Biol. Macromol. 2008, 42, 483-490.

14. Dill, K.A.; Shortie, D. Denatured states of proteins. Ann. Rev. Biochem. 1991, 60, 795-825.

15. Petitpas, I.; Petersen, C.E.; Ha, C.E.; Bhattacharay, A.A.; Zunszain, P.A.; Ghuman, J. Structural basis of albumin-thyroxine interactions and familial dysalbuminemic hyperthyroxinemia. Proc. Natl. Acad. Sci. USA 2003, 100, 6440-6445.

16. Petitpas, I.; Grune, T.; Bhattacharya, A.A.; Curry, S. Crystal structures of human serum albumin complexed with monounsaturated and polyunsaturated fatty acids. J. Mol. Biol. 2001, 314, 955-960.

17. Zemek, F.; Korabecny, J.; Sepsova, V.; Karasova, J.Z.; Musilek, K.; Kuca, K. Albumin and a1-acid glycoprotein: Old acquaintances. Expert Opin. Drug Metab. Toxicol. 2013, 9, 943-954.

18. Bajgar, J. Optimal choice of acetylcholinesterase reactivators for antidotal treatment of nerve agent intoxication. Acta Medica 2010, 53, 207-211.

19. Colovic, M.B.; Krstic, D.Z.; Lazarevic-Pasti, T.D.; Bondzic, A.M.; Vasic, V.M. Acetylcholinesterase inhibitors: Pharmacology and toxicology. Curr. Neuropharmacol. 2013, 11, 315-335.

20. Litchfield, M.H. Estimates of acute pesticide poisoning in agricultural workers in less developed countries. Toxicol. Rev. 2005, 24, 271-278.

21. Karasova, J.Z.; Chladek, J.; Hroch, M.; Josef, F.; Hnidkova, D.; Kuca, K. Pharmacokinetic study of two acetylcholinesterase reactivators, trimedoxime and newly synthesized oxime K027, in rat plasma. J. Appl. Toxicol. 2013, 33, 18-23.

22. Kalasz, H.; Szegi, P.; Janoki, G.; Balogh, L.; Postenyi, Z.; Musilek, K.; Petroianu, G.A.; Siddiq, A.; Tekes, K. Study on medicinal chemistry of K203 in wistar rats and beagle dogs. Curr. Med. Chem. 2013, 20, 2137-2144.

23. Karasova, J.Z.; Novotny, L.; Antos, K.; Zivna, H.; Kuca, K. Time-dependent changes in concentration of two clinically used acetylcholinesterase reactivators (HI-6 and obidoxime) in rat plasma determined by HPLC techniques after in vivo administration. Anal. Sci. 2010, 26, 63-67.

24. Kassa, J.; Misik, J.; Karasova, J.Z. A comparison of the potency of a novel bispyridinium oxime K203 and currently available oximes (obidoxime, HI-6) to counteract the acute neurotoxicity of sarin in rats. Basic Clin. Pharmacol. Toxicol. 2012, 111, 333-338.

25. Karasova, J.Z.; Pohanka, M.; Pavlik, M.; Kassa, J.; Musilek, K.; Zemek, F.; Kuca, K. Distribution study of acetylcholinesterase reactivators after application of therapeutic doses in guinea pigs. Toxicol. Lett. 2011, 205, S116. 
26. Kuca, K.; Musilek, K.; Jun, D.; Pohanka, M.; Ghosh, K.K.; Hrabinova, M. Oxime K027: Novel low-toxic candidate for the universal reactivator of nerve agent-and pesticide-inhibited acetylcholinesterase. J. Enzyme Inhib. Med. Chem. 2010, 25, 509-512.

27. Matos, K.S.; da Cunha, E.F.F.; Goncalves, A.S.; Wilter, A.; Kuca, K.; Franca, T.C.C.; Ramalho, T.C. First principles calculations of thermodynamics and kinetic parameters and molecular dynamics simulations of acetylcholinesterase reactivators: Can mouse data provide new insights into humans? J. Biomol. Struct. Dyn. 2012, 30, 546-558.

28. Worek, F.; Thiermann, H. Reactivation of organophosphate-inhibited human acetylcholinesterase by isontrosoacetone (MINA): A kinetic analysis. Chem. Biol. Interact. 2011, 194, 91-96.

29. Skovira, J.W.; O’Donnell, J.C.; Koplovitz, I.; Kan, R.K.; McDonough, J.H.; Snih, T.M. Reactivation of brain acetylcholinesterase by monoisonitrosoacetone increases the therapeutic efficacy against nerve agents in guinea pigs. Chem. Biol. Interact. 2010, 187, 318-324.

30. Kovarik, Z.; Macek, N.; Sit, R.K.; Radic, Z.; Fokin, V.V.; Baarry Sharpless, K.; Taylor, P. Centrally acting oximes in reactivation of tabun-phosphoramidated AChE. Chem. Biol. Interact. 2013, 203, 77-80.

31. Karasova, J.Z.; Kassa, J.; Jung, Y.S.; Musilek, K.; Pohanka, M.; Kuca, K. Effect of several new and currently available oxime cholinesterase reactivators on tabun-intoxicated rats. Int. J. Mol. Sci. 2008, 9, 2243-2252.

32. Kassa, J.; Karasova, J.Z.; Tesarova, S.; Kuca, K.; Musilek, K. A comparison of the ability of newly-developed bispyridinium oxime K203 and currently available oximes (trimedoxime, obidoxime, HI-6) to counteract the acute neurotoxicity of soman in rats. Toxicol. Mech. Methods 2010, 20, 445-451.

(C) 2013 by the authors; licensee MDPI, Basel, Switzerland. This article is an open access article distributed under the terms and conditions of the Creative Commons Attribution license (http://creativecommons.org/licenses/by/3.0/). 